\title{
Shear Driven Turbulence and Coherent Structures in Solar Surface Simulations
}

\begin{abstract}
F. Kupka
Max-Planck-Institute for Astrophysics, Karl-Schwarzschild Str. 1, D-85748 Garching, Germany email: fk@mpa-garching.mpg.de

Abstract. Numerical simulations of convection near the solar surface are now advanced enough to reproduce both a large set of observational data and provide tests for convection models. We discuss the role of coherent structures in models of solar p-mode excitation, for which the analysis of numerical simulations has provided key inputs in the modelling. The robustness of these simulations is shown by a comparison illustrating the influence of boundary conditions on ensemble averaged quantities. In a concluding example advanced high resolution simulations are shown to resolve the onset of shear driven turbulence generated by up- and downflow structures.
\end{abstract}

Keywords. Convection, turbulence, Sun: interior, Sun: granulation, Sun: helioseismology

\section{Some astrophysical constraints imposed by solar convection}

A brief look on observations of solar surface convection by means of imaging spectroscopy and narrow band photometry (Title 2007, e.g.) shows the well-known pattern of bright patches (granules) embedded in a network of dark regions (intergranular lanes). This structure dominates the solar surface outside regions of enhanced magnetic activity. Analysis of spectroscopic data and corresponding numerical simulations of these structures has demonstrated that granules are upflow regions of gas which is hotter than its surrounding networks which in turn are created by regions of cold downflows. Since the granules cover a larger fraction of the surface than the intergranular network, mass conservation requires the upflows to have lower average velocities than their downflow counterparts (for a review see Spruit, Nordlund \& Title 1990).

At current instrumental resolutions of about $100 \mathrm{~km}$ (Scharmer, Gudiksen, Kiselman, et al. 2002; Title 2007; Kosugi, Matsuzaki, Sakao, et al. 2007) the solar surface granulation looks like a laminar flow, as is also found in numerical simulations at somewhat higher resolution of $20 \mathrm{~km}$ to $50 \mathrm{~km}$ (see Stein \& Nordlund 1998; Robinson, Demarque, Li, et al. 2003; Wedemeyer, Freytag, Steffen, et al. 2004; Vögler, Shelyag, Schüssler, et al. 2005; Steffen 2007). Are these surface layers representative for the entire solar convection zone? Not necessarily. Granules can cool off by radiating into space, while hot flows inside the Sun are surrounded by optically thick fluid, which increases the cooling time scale. More importantly, the solar surface layers are highly stratified. The local (pressure) scale height $H_{p}=P /(\rho g)$ increases by more than two orders of magnitudes from the top to the bottom of the solar convection zone, because the pressure $P$ increases much faster towards the interior than the product of density $\rho$ and (local) gravitational acceleration $g$. As can be seen from classical and current solar structure models published in textbooks (Stix 1989 or Weiss, Hillebrandt, Thomas, et al. 2004, e.g.), a density contrast of $\sim 625,000: 1$ and a temperature contrast of $\sim 350: 1$ is found between the bottom and the top of the solar convection zone. Since $g$ changes by less than a factor of 2 (decreasing towards the top of the convection zone), which coincidentally is compensated by a similar change in mean molecular weight $\mu$ (increasing towards the top due to hydrogen 
becoming neutral), $H_{p}$ is $\sim 50,000 \mathrm{~km}$ near the bottom of the solar convection zone while it is $\sim 150 \mathrm{~km}$ near its top. Similar holds for surface convection zones in cool main sequence stars other than the Sun. Finally, the flow speed as measured in Mach numbers is about 0.3 near the surface and as low as $10^{-4}$ close to the bottom of the solar convection zone (cf. also Stix 1989). Consequently, granules experience a very strong decompression in the solar photosphere which has no counterpart in the solar interior.

Taking mass conservation into account and assuming that horizontal flows are mostly subsonic one can estimate (Stein \& Nordlund 1998, e.g.) that horizontal scales $L$ are limited by $L \lesssim 4 H_{p} /$ Ma. Here, Ma is the vertical flow Mach number $(<1)$, the vertical flow velocity in units of local sound speed. Thus, at the solar surface the granule diameters $D \sim L$ are less than about $2000 \mathrm{~km}$, in agreement with observations which put $D$ in the range of $1200 \mathrm{~km}$ (see Chap. 1.3 and 6.3 in Stix 1989). Consequently, current simulations of surface granulation cannot include the entire solar convection zone and have to be restricted to a "box-in-a-star" approximation (see also Freytag, Steffen \& Dorch 2002). The other way round a simulation of the bottom of the solar convection zone cannot account for surface granulation, since it necessarily has to be global, if the expected energy carrying scales (of order $H_{p}$ ) should be represented in the simulation. For stars with expanded atmospheres the situation is more in favour of global simulations, since $H_{p}$ at the stellar surface becomes large, as it scales with $1 / g$, which makes a "star-in-a-box" approach feasible for simulations of their surface convection even with current high performance computers (see again Freytag, Steffen \& Dorch 2002). Tests based on spectroscopy and helioseismology have given numerical simulations of solar and stellar surface convection strong observational support (see the above references and further observational tests as presented, for instance in Rosenthal, Christensen-Dalsgaard, Nordlund, et al. 1999 and in Asplund, Nordlund, Trampedach, et al. 2000) and thus corroborated the "box-in-a-star" approach for the case of (geometrically) thin stellar atmospheres.

\section{Numerical simulations of solar and stellar surface convection}

Numerical simulations of solar and stellar surface convection compute volume averages of the basic variables $\rho, \rho \boldsymbol{u}$, and $\rho e$, the densities of mass, momentum, and total energy (with the latter defined as the sum of kinetic and thermal energy). Hence, in a terminology borrowed from engineering sciences, they are large eddy simulations (LES). On their numerical grid LES represent the large spatial scales where most of the kinetic energy is carried. For stellar surface convection the scales on which radiative cooling occurs have to be included, too, which can be achieved with current computational resources. Length scales in the flow smaller than the mesh width of the grid are accounted for by some subgrid scale model (or other approximations), while scales larger than the box size (grid extent) assumed in the simulation are handled by the boundary conditions. The simulations start from "typical" initial conditions and proceed through a relaxation phase until quasi-stationarity of key variables is found, such as time averaged total energy flux and root mean square velocities as a function of depth. For a discussion of relaxation of such simulations see Chan \& Sofia (1986) and Kupka (2008). From that point onwards a statistical interpretation of the simulation results can be performed. Time integrations of horizontal averages are used to generate ensemble averages which can be compared to quantities used in stellar structure and evolution computations and to stellar observations (which are averages over time and, usually, over the stellar disk, too). Other tools include graphical visualization of the flow, particularly of three-dimensional features, by means of volume rendering and animations to study their development in time. 
Unresolved scales cannot be avoided in numerical simulations of stellar convection, as non-linear interactions caused by advection processes become very large in stellar environments and hence dominate over viscous processes. This is expressed by a large Reynolds number $\operatorname{Re}=U L / \nu$, which compares length scales and velocities $L$ and $U$ of the energy carrying scales of the flow (for instance, the solar granules and their average velocity) with the kinematic viscosity $\nu$. For solar granules, $L \sim D, U(L) \sim 2 \ldots 3 \mathrm{~km} \mathrm{~s}^{-1}$, and $\nu \sim 1740 \mathrm{~cm}^{2} \mathrm{~s}^{-1}$, where we take $U(L)$ from spectroscopy and numerical simulations (cf. Asplund, Nordlund, Trampedach, et al. 2000) and $\nu$ from Cowley (1990) (Tables 1 and 2 for a layer at the surface with $\mathrm{T}=5660 \mathrm{~K}$, with values for $\mu=\nu \rho$ based on Edmonds 1957). Thus, Re $\sim 10^{10}$ and advection completely dominates over viscous friction. This justifies modelling solar surface convection as a nearly inviscid flow. Assuming a Kolmogorov scaling for length scales $l \ll L$ one can estimate dissipation by viscous friction to occur at $l_{d}$ where $L / l_{d} \sim \operatorname{Re}^{3 / 4}$ (cf. Lesieur 1997), thus $L / l_{d} \sim 10^{7.5}$. Hence, on an equispaced spatial grid $N>10^{22}$ points are required to resolve $l_{d}$. This is neither affordable, nor necessary, since most of the observed physics takes place at scales $l \sim L$. The assumption behind choosing a particular grid size $h \gg l_{d}$ is based on the expectation that the dynamically important processes take place for $l>h$ and thus, averages over scales $l \leqslant h$ can be represented by hyperviscosity, or non-linear numerical viscosity, or turbulent viscosity, combined with various treatments of shocks (for a discussion of choosing those scales in the context of stellar convection simulations see Kupka 2008).

\section{Convection models and coherent structures}

Coherent structures are defined as spatial regions that at a given time show some organization with respect to any quantity related to the flow (Lesieur 1997). The basic concept and its role in turbulent flows is already described in Townsend (1956) and even earlier references can be found in the literature (see also the reviews by Cantwell 1990 and Narasimha 1990). The up- and downflow pattern evident in convective flows in general and in stellar surface convection in particular is just one more example. It is instructive to compare the analysis of Stein \& Nordlund (1998) with respect to the distribution of hot and cold areas in up- and downflows with measurements of convection in the atmosphere of the Earth, the planetary boundary layer, in Hartmann, Kottmeier \& Raasch (1997) and Hartmann, et al. (1999). Clearly, the role of up- and downflows is reversed in the sense that upflows cover a smaller area in the planetary boundary layer than the downflows (which is usually explained by driving convection through heating at the bottom instead of cooling at the top). Moreover, the averages for the solar surface have to be taken over horizontal areas of equal optical Rosseland depth instead of fixed geometrical height to avoid that strong large scale fluctuations introduced by the granules themselves skew the statistical distribution under investigation. Taking that into account the distributions of velocity and temperature fluctuations around their horizontal mean look quite similar. Could that be used in convection models developed for both systems?

In Kupka \& Muthsam (2007b) fluxes of velocity and temperature fluctuations as obtained from various convection models were tested with numerical simulations for cases of both inefficient convection (where the radiative flux still transports most of the energy, as, for instance, in surface convection in A-stars of intermediate temperature) and efficient convection. It was noted that for the case of efficient convection only a closure model explicitly accounting for the skewness of both velocity and temperature fluctuations gave satisfactory results when compared to the simulations (see also Kupka 2007). Previous models had relied on a diffusion approach (Xiong 1978) for third order moments 
(ensemble averages of products of fluctuations of the basic field variables relative to their mean), the quasi-normal approximation for fourth order moments (Canuto 1992, Canuto 1993), or used the eddy damping approach to improve over the latter (Canuto, Cheng \& Howard 2001). None of these models explicitly accounts for the coherent, large scale nature of convective flows. This was concluded to be one of the major shortcomings in the previous models, when applied to the case of deep zones of efficient convection (Kupka \& Muthsam 2007a-c and Kupka 2007).

In Gryanik \& Hartmann (2002) and Gryanik, et al. (2005) it was suggested that the spatial asymmetry in the distribution of temperature and velocity fields inside a convection zone should be accounted for through a model which interpolates between the two cases of large and small skewness. In the first case, the model yields the same result as a two-scale mass-flux model (Gryanik \& Hartmann 2002) which consists of averaging separately the velocity and temperature fields over columns defined by the locations where the difference between each field and its horizontal average changes sign. Fluctuations within the up- or downflow, or among different columns defined this way, are neglected in that limit. In the small skewness limit the model recovers the quasi-normal approximation. A completely different approach to account for the non-Gaussian nature of convective flows was suggested by Cheng, Canuto \& Howard (2005), where the different terms appearing in the dynamical equations for third order moments (Canuto 1993, Canuto, Cheng \& Howard 2001) are used to model the contributions neglected by the quasi-normal approach to fourth order moments. As explained in Canuto (2007), this yields a Reynolds stress model with properties very similar to plume models and traditional versions of mass-flux models. The model was found to be in much better agreement with observations of the planetary boundary layer than its predecessors.

Kupka \& Robinson (2007) showed that the model of Gryanik \& Hartmann (2002) and of Gryanik, et al. (2005) provides improvements by up to an order of magnitude compared to the quasi-normal approximation in consistency tests with numerical simulations for surface convection in the Sun and in a K dwarf. They corroborated the results previously found for the planetary boundary layer and later on confirmed for the case of convection in the ocean (Losch 2004). Encouraged by this wide range of applicability the model was extended in Belkacem, et al. (2006a) to account for the turbulent fluctuations within the downdrafts by a plume model. In this variant the model was applied in computations of excitation rates of solar p-modes (Belkacem, et al. 2006b). Mode excitation occurs due to shear stresses and entropy fluctuations (cf. also Samadi \& Goupil 2001), thus the power injected into a p-mode depends quadratically on the vertical velocity field, and as a consequence of mass conservation a skewed vertical velocity field provides a higher mode excitation rate than its unskewed counterpart. The new model for p-mode excitation rates provides an improvement by an order of magnitude over previous models (local convection models without turbulent pressure and assuming a quasi-normal distribution of fourth order moments with zero skewness of velocity and temperature fields). Model predictions now agree with observations to within measurement uncertainties. In Samadi, et al. (2008) it was shown that the model also recovers the p-mode excitation rates derived for $\alpha$ Cen A. Although observations for this star are not yet accurate enough to distinguish between the inclusion of skewness and its neglect in the models, it was clearly shown that convection models must account for turbulent pressure to agree with observations and in addition that both skewness and a deviation of temporal fourth order correlations from a normal distribution can be probed with sufficiently accurate observations from asteroseismology. It should be pointed out here that probing a convection model with p-mode excitation rates is a much more stringent test than using just the p-mode frequencies, since the latter can be recovered with 
different temperature-pressure profiles of the surface layers of a solar (or stellar) model. The classical requirement of recovering the solar radius of the present Sun is an even weaker test, since integral quantities can be reproduced with many different internal thermal and hydrostatical structure profiles, as long as there is an adjustable parameter in the convection model and, say, an unknown helium content (to match the solar luminosity).

\section{Comparison of numerical simulations among each other}

How reliable are numerical simulations of solar surface convection for probing convection models? In addition to testing the simulations with observations (cf. Sect. 1), it is instructive to compare the predictions for statistical correlations by the different codes for various resolutions and boundary conditions. This could corroborate the numerical methods used in the computations of the flow and elucidate possible weaknesses of the simulations. It would demonstrate to what extent the flow field is influenced by the boundary conditions and the domain size chosen. The role of non-grey radiative transfer and the influence of different numerical methods and viscosity models on the large scale, coherently structured flow could be studied, too. In the following we discuss such a comparison with results that have kindly been provided by M. Steffen $\left(\mathrm{CO}^{5} \mathrm{BOLD}\right.$ code, Freytag, Steffen \& Dorch 2002) and F.J. Robinson (CKS code, Chan \& Sofia 1996, Kim \& Chan 1998). The $\mathrm{CO}^{5} \mathrm{BOLD}$ code is based on a Roe-scheme and combines a non-linear numerical viscosity with a model for subgrid-scale viscosity (SmagorinskyLilly model, Smagorinsky 1963). Equation of state and opacity in this code are taken from standard model atmosphere codes (Kurucz 1979, Gustafsson, et al. 1975, and more recent versions). The CKS code combines a conservative scheme with the same explicit subgrid-scale viscosity as used in the $\mathrm{CO}^{5} \mathrm{BOLD}$ code, but additionally that viscosity term is boosted around shocks in the flow to handle the latter. Opacities and equation of state are the same which are commonly used in stellar structure and evolution models (Iglesias \& Rogers 1996, Rogers, Swenson \& Iglesias 1996, Alexander \& Ferguson 1994). We compare results from two different runs with each code. All runs are based on the "box-in-a-star" approach with a Cartesian geometry, periodic horizontal boundary conditions, and a constant vertical gravitational acceleration set equal to the solar one. For the simulations with the $\mathrm{CO}^{5} \mathrm{BOLD}$ code, the vertical boundary conditions are open (Steffen 2007 and priv. comm.). The high resolution case features a 5-bin non-grey radiative transfer, a grid of $400 \times 400 \times 165$ points with a constant horizontal resolution of $28 \mathrm{~km}$ and a variable vertical one of $12 \mathrm{~km}$ to $28 \mathrm{~km}$. The resulting simulation box has a volume of $11.2 \times 11.2 \times 3.1 \mathrm{Mm}^{3}$. The deep simulation case assumes grey radiative transfer, a grid of $200 \times 200 \times 250$ points with a constant horizontal resolution of $56 \mathrm{~km}$ and a constant vertical one of $21 \mathrm{~km}$. The simulation box has a volume of $11.2 \times 11.2 \times 5.2 \mathrm{Mm}^{3}$ in this case. For both simulations with the CKS code, closed vertical boundary conditions are imposed (free slip, with a constant energy input flux corresponding to the solar effective temperature). Radiative transfer is treated in a grey, 3D Eddington approximation. The first simulation is "model D" from Robinson, Demarque, Li, et al. (2003), also used in Kupka \& Robinson (2007). It assumes a grid with $58 \times 58 \times 170$ points with a constant resolution of $50 \mathrm{~km}$ horizontally and $17.6 \mathrm{~km}$ vertically which result in a box volume of $2.9 \times 2.9 \times 3 \mathrm{Mm}^{3}$. The second simulation (F.J. Robinson 2007, priv. comm.) has $117 \times 117 \times 190$ points with a constant resolution of $35 \mathrm{~km}$ horizontally and $15 \mathrm{~km}$ vertically which result in a box volume of $4.1 \times 4.1 \times 2.835 \mathrm{Mm}^{3}$. Most importantly, the vertical location of the simulation box relative to the optical surface (with $\tau_{\text {ross }} \approx 1$ ) has been shifted further upwards by about $150 \mathrm{~km}$ compared to the "model D" 

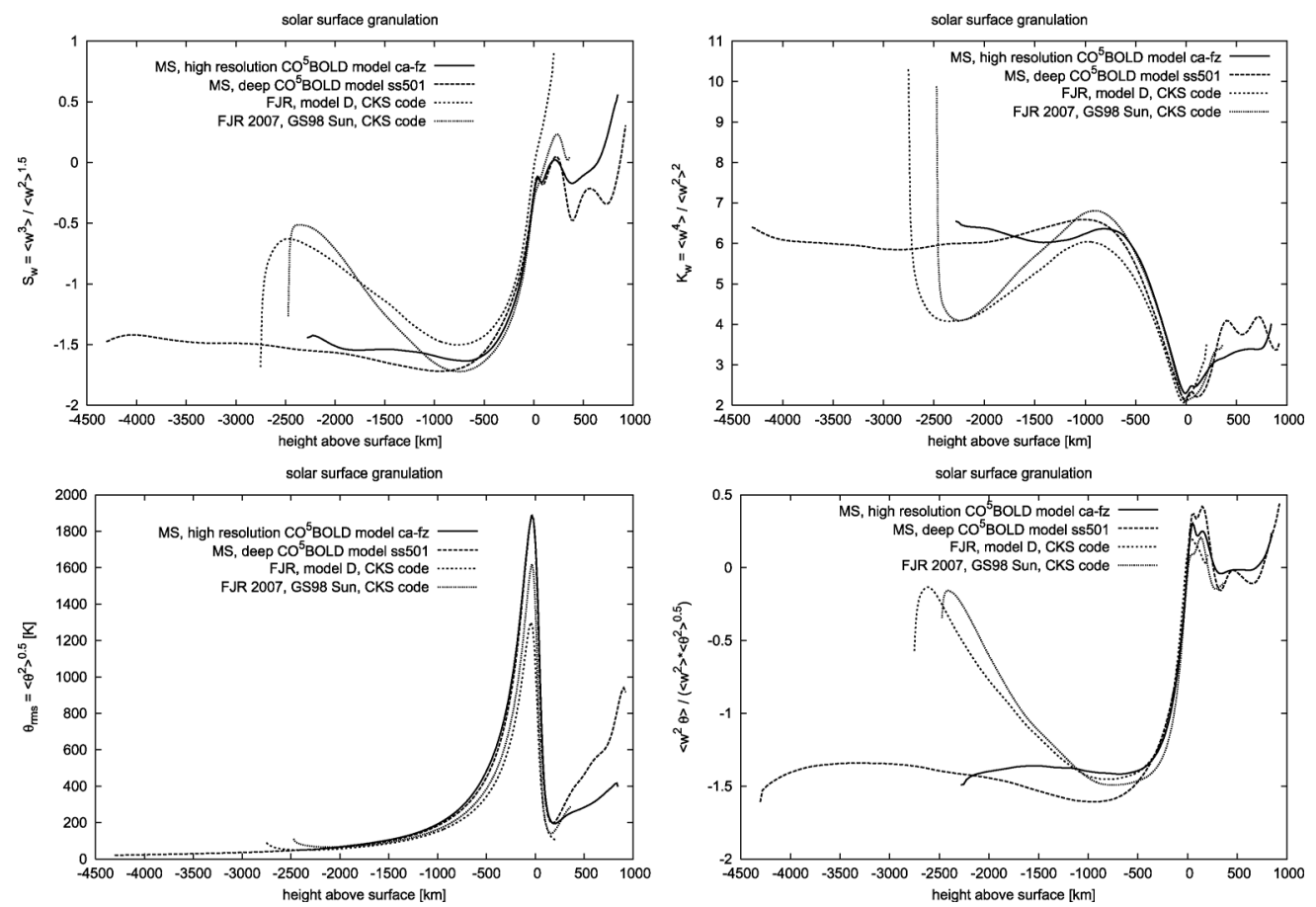

Figure 1. Comparison of simulations with open boundary conditions (data kindly provided by M. Steffen and denoted MS) and with closed boundary conditions (data kindly provided by F.J. Robinson and denoted FJR). Skewness and kurtosis of vertical velocity fluctuations ( $S_{w}$, $K_{w}$ ), the root mean square of temperature fluctuations (in $[\mathrm{K}]$ ), and a normalised cross-correlation, $\overline{w^{2} \theta} /\left(\overline{w^{2}}\left(\overline{\theta^{2}}\right)^{0.5}\right)$ are shown.

simulation. This significantly reduces the influence of the upper boundary, as follows from the comparison of various ensemble averages from all four simulation runs presented in Fig. 1. The data are given as a function of height (with a zero-point chosen to be the solar surface at $\tau_{\text {ross }} \sim 1$ in "model D" while data from other simulations are plotted with a constant vertical shift applied to their own measure of height such that just below the surface the average temperature agrees among the simulations shown). The quantities have been computed from a time average of the horizontal averages of the local fluctuations of velocity and temperature (which were taken relative to their horizontal average at each point in time included in the averaging). The influence of closed vertical boundary conditions extend to between 1.5 and 2.5 pressure scale heights, as discussed in Kupka (2008). Since the boxes considered are sufficiently deep, all four simulations agree quite well between the surface at $0 \mathrm{~km}$ to about $1000 \mathrm{~km}$ further below. This range includes the entire superadiabatic layer and the top of the quasi-adiabatic interior of the solar convection zone. Compared to $K_{w}$ and $\overline{w^{2} \theta} /\left(\overline{w^{2}}\left(\overline{\theta^{2}}\right)^{0.5}\right)$ the skewness of vertical velocity, $S_{w}$, is more sensitive to the (upper) vertical boundary condition. Pushing that boundary sufficiently upwards, as done in the more recent model computed with the CKS code (FJR 2007), significantly improves the comparison with the $\mathrm{CO}^{5}$ BOLD simulations, which both have open upper boundary conditions (located even further upwards). Moving the closed boundary further upwards also allows for larger temperature fluctuations which again are in better agreement with the simulations with open boundary conditions. 


\section{Simulations of fully turbulent solar convection}

At the bottom of the solar photosphere Re $\sim 10^{10}$, as mentioned in Sect. 1, while the Prandtl number Pr, the ratio of kinematic viscosity and thermometric conductivity, remains of order $\sim 10^{-9}$. Interestingly, the product of both, the Peclet number $\mathrm{Pe}=$ $\mathrm{Re} \cdot \mathrm{Pr}$, is hence of order 10. Consequently, it is possible to model radiative cooling at the solar surface realistically (without resorting to artificially enhanced conductivities, as is necessary for convection simulations of the lower part of the solar convection zone, where Pe exceeds $10^{6}$ ). Since up- and downflows in solar surface convection move relative to each other at almost sonic speed, one would expect large shear stresses to occur and a large acoustic flux to be created in the layers underneath the visible surface, which are no longer affected by strong radiative cooling. What resolution would be required to resolve the turbulence generated by the Kelvin-Helmholtz shear instability? If we estimate the energy carrying scale to be of the order of the granule diameter $L \sim D$ and assume a Kolomogorov scaling (cf. Sect. 2), we obtain that the grid size $h$ should be of the order of $4 \mathrm{~km}$ to achieve an effective Reynolds number of about 2300 (Kupka 2008). In that case the shear driven turbulence is at least partially resolved on the computational grid. This kind of scaling is different in 2D (Kupka 2008), because conservation of vorticity along streamlines (cf. Lesieur 1997) reduces the number of internal degrees of freedom in the flow, and permits access to a different dynamical range in comparison with a 3D simulation. Thus, if properties related to turbulence in the flow are of interest in a simulation, 3D simulations are necessary not only quantitatively but also qualitatively.

To reduce the influence of periodic horizontal boundary conditions and in particular to minimize spurious "self-interactions" of granules in a "box-in-a-star" simulation it is advisable to consider a simulation volume that is large enough to hold on average up to half a dozen up- and downflow structures along any given horizontal direction. At a resolution of $5 \mathrm{~km}$ this would require about $1500^{3}$ grid points. An alternative to such an expensive calculation is the use of local grid refinement. This permits to consider a more limited domain which only contains perhaps up to three up- and downflow structures along any given horizontal direction at the desired resolution. This high resolution domain is embedded in a larger simulation box with a lower spatial resolution. The grid refinement efficiently reduces the influence of boundary conditions except for those due to more laminar flow structures which occasionally enter the high resolution domain. An example for this strategy can be found in Muthsam, Löw-Baselli, Obertscheider, et al. (2007) who performed 2D simulations of solar surface convection at resolutions of better than $3 \mathrm{~km}$ based on this approach with the ANTARES simulation code. The simulations very clearly revealed Kelvin-Helmholtz instabilities due to shear between up- and downflows which in turn created all the flow structures expected for a turbulent flow in two spatial dimensions. Remarkably, the solar photospheric layers remained smooth, apart from waves and shock fronts occasionally entering from below. Since the code uses (among other options) a high resolution ( $5^{\text {th }}$ order) WENO scheme (Liu, Osher \& Chan 1994), it does not have to rely on artificial diffusion schemes once a threshold resolution (somewhat larger than $50 \mathrm{~km}$ horizontally in the solar case) is exceeded (Muthsam, LöwBaselli, Obertscheider, et al. 2008). Thus, the onset of shear driven turbulence can be observed at lower resolution than with previously used methods (see also Kupka 2008).

What happens in such simulations in 3D? In Fig. 2 we show results from a simulation at $7.1 \times 9.8 \times 9.8 \mathrm{~km}^{3}$ resolution (first co-ordinate denotes the vertical direction) of $259 \times 425 \times 393$ cells (and thus a volume of $2.8 \times 4.2 \times 3.8 \mathrm{Mm}^{3}$ ) embedded in a region with a resolution twice lower vertically and four times lower horizontally. It fills a volume of $2.8 \times 11.2 \times 11.2 \mathrm{Mm}^{3}$ and is described in more detail in Muthsam, Löw-Baselli, 


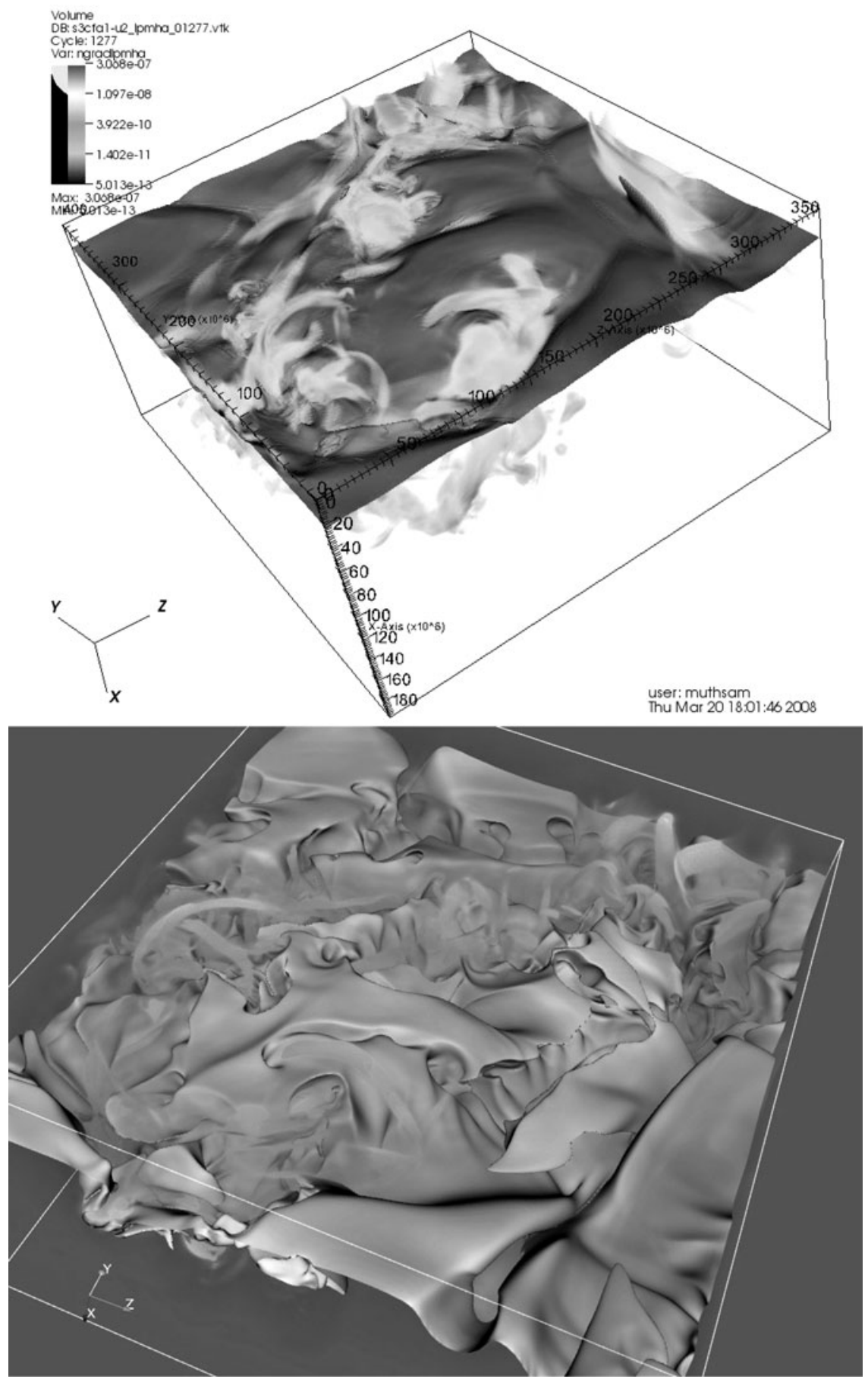

Figure 2. 3D high resolution simulations of shear driven turbulence in solar surface convection. The upper panel shows a snapshot of an isosurface at $T=6000 \mathrm{~K}$ with a volume rendering of strong local pressure fluctuations. The lower panel shows an isosurface at $T=8000 \mathrm{~K}$ at the same point in time (note the different viewing angle; volume rendering is less discernible). 
Obertscheider, et al. (2008). The figure shows a decaying granule and its surroundings at two different depth levels: at the visible surface (upper panel) and underneath. At visible layers the isosurface still looks quit smooth and quasi-laminar. Below those surface layers, however, the simulation appears highly turbulent (lower panel). As pointed out in Muthsam, Löw-Baselli, Obertscheider, et al. (2008), at a resolution of close to $10 \mathrm{~km}$, similar to the highest resolution case of Vögler \& Schüssler (2007), the numerical scheme is still essential to track the onset of turbulence, which is less prominent and delayed to occur at higher resolution with a numerical method which requires a higher viscosity.

\section{Summary and outlook}

Numerical simulations have confirmed the role of coherent structures in astrophysical systems which had previously been inferred from observational data. They are now advanced enough to probe the advection properties of non-local convection models used to study solar p-mode excitation. The latter have shown that it is necessary to explicitly account for organised motion in convection modelling. In turn, direct comparisons of statistical properties among different numerical simulations allow quantifying the influence of boundary conditions and other physical simplifications and demonstrate the strengths and limitations of individual simulation codes. Recent simulations have shown how turbulence can be present in a flow despite being shrouded from direct observations, as is the case in solar surface convection. Future improvements in numerical techniques and computing capabilities will allow resolving shear driven turbulence for an even larger number of astrophysical problems and help in developing a more refined physical picture of turbulent flows in astrophysics. The simulations will continue to complement observations by providing another source of test data for convection models which in turn can be further refined as required by their expected continuous use in calculations of stellar structure and evolution for some time to come.

\section{Acknowledgements}

This work is partially supported by the DFG subproject KU 1954/3-1 of SPP 1276/1. The author is grateful to M. Steffen and F. J. Robinson for providing the data used for Fig. 1 and to H. J. Muthsam for providing the colour images used to produce Fig. 2.

\section{References}

Alexander, D. R. \& Ferguson, J. W. 1994, ApJ 437, 879

Asplund, M., Nordlund, Å., Trampedach, R., Allende Prieto, C., \& Stein, R. F. 2000, A\&্A 359, 729

Belkacem, K., Samadi, R., Goupil, M. J., \& Kupka, F. 2006a, A\&A 460, 173

Belkacem, K., Samadi, R., Goupil, M. J., Kupka, F., \& Baudin, F. 2006b, A\&\&A 460, 183

Cantwell, B. 1990, in J. L. Lumley (ed.), Whither Turbulence? Turbulence at the Crossroads, Springer Lecture Notes in Physics, (Berlin: Springer-Verlag), vol. 357, p. 97

Canuto, V. M. 1992, ApJ 392, 218

Canuto, V. M. 1993, ApJ 416, 331

Canuto, V. M. 2007, in F. Kupka, I. W. Roxburgh \& K. L. Chan (eds.), Proc. IAU S 239, (Cambridge: Camb. Univ. Press), p. 19

Canuto, V. M., Cheng, Y., \& Howard, A. 2001, J. Atmos. Sci. 58, 1169

Chan, K. L. \& Sofia, S. 1986, ApJ 307, 222

Chan, K. L. \& Sofia, S. 1996, ApJ 466, 372

Cheng, Y., Canuto, V. M., \& Howard, A. M. 2005, J. Atmos. Sci. 62, 2189

Cowley, C. R. 1990, ApJ 348, 328 
Edmonds, F. N. 1957, ApJ 125, 535

Freytag, B., Steffen, M., \& Dorch, B. 2002, Astron. Nachrichten 323, 213

Gryanik, V. M. \& Hartmann, J. 2002, J. Atmos. Sci. 59, 2729

Gryanik, V. M., Hartmann, J., Raasch, S., \& Schröter, M. 2005, J. Atmos. Sci. 62, 2632

Gustafsson, B., Bell, R. A., Eriksson, K., \& Nordlund, A. 1975, A\& A 42, 407

Hartmann, J., Kottmeier, C., \& Raasch, S. 1997, Bound.-Layer Meteor. 84, 45

Hartmann, J., et al. 1999, Polar Research Rep., (Bremerhaven: Alfred Wegener Institute for Polar and Marine Sciences), vol. 305, p. 81

Iglesias, C. A. \& Rogers, F. J. 1996, ApJ 464, 943

Kim, Y.-C. \& Chan, K. L. 1998, ApJ (Letters) 496, L121

Kosugi, T., Matsuzaki, K., Sakao, T., Shimizu, T., Sone, Y., Tachikawa, S., Hashimoto, T., Minesugi, K., Ohnishi, A., Yamada, T., Tsuneta, S., Hara, H., Ichimoto, K., Suematsu, Y., Shimojo, M., Watanabe, T., Shimada, S., Davis, J. M., Hill, L. D., Owens, J. K., Title, A. M., Culhane, J. L., Harra, L. K., Doschek, G. A., \& Golub, L. 2007, Solar Phys. 243, 3

Kupka, F. 2007, in: F. Kupka, I. W. Roxburgh \& K. L. Chan (eds.), Proc. IAU S 239, (Cambridge: Camb. Univ. Press), p. 92

Kupka, F. 2008, in: W. Hillebrandt, F. Kupka (eds.), Interdisciplinary Aspects of Turbulence, Springer Lecture Notes in Physics, (Berlin: Springer-Verlag), in print

Kupka, F. \& Muthsam, H. J. 2007a, in: F. Kupka, I. W. Roxburgh \& K. L. Chan (eds.), Proc. IAU S 239, (Cambridge: Camb. Univ. Press), p. 80

Kupka, F. \& Muthsam, H. J. 2007b, in: F. Kupka, I. W. Roxburgh \& K. L. Chan (eds.), Proc. IAU S 239, (Cambridge: Camb. Univ. Press), p. 83

Kupka, F. \& Muthsam, H. J. 2007c, in: F. Kupka, I. W. Roxburgh \& K. L. Chan (eds.), Proc. IAU S 239, (Cambridge: Camb. Univ. Press), p. 86

Kupka, F. \& Robinson, F. 2007, MNRAS 374, 305

Kurucz, R. L. 1979, ApJS 40, 1

Lesieur, M. 1997, Turbulence in Fluids, 3rd ed., (Dordrecht: Kluwer Academic Publishers)

Liu, X., Osher, S., \& Chan, T. 1994, J. Comput. Phys. 115, 200

Losch, M. 2004, Geophys. Res. Lett. 31, L23301

Muthsam, H. J., Löw-Baselli, B., Obertscheider, Chr., Langer, M., Lenz, P., \& Kupka, F. 2007, MNRAS 380, 1335

Muthsam, H. J., Löw-Baselli, B., Obertscheider, Chr., Langer, M., Lenz, P., \& Kupka, F. 2008, to be submitted

Narasimha, R. 1990, in J. L. Lumley (ed.), Whither Turbulence? Turbulence at the Crossroads, Springer Lecture Notes in Physics, (Berlin: Springer-Verlag), vol. 357, p. 13

Robinson, F. J., Demarque, P., Li, L. H., Sofia, S., Kim, Y.-C., Chan, K. L., \& Guenther, D. B. 2003, MNRAS, 340, 923

Rogers, F. J., Swenson, F. J., \& Iglesias, C. A. 1996, ApJ 456, 902

Rosenthal, C. S., Christensen-Dalsgaard, J., Nordlund, Å., Stein, R. F., \& Trampedach, R. 1999, $A \mathscr{E} A 351,689$

Samadi, R. \& Goupil, M.-J. 2001, A\& A 370, 136

Samadi, R., Belkacem, K., Goupil, M. J., Dupret, M.-A., \& Kupka, F. 2008, A\& $\&$, in print

Scharmer, G. B., Gudiksen, B. V., Kiselman, D., Löfdahl, M. G., \& Rouppe van der Voort, L. H. M. 2002, Nature 420, 151

Smagorinsky, J. 1963, Mon. Weather Rev. 91, 99

Spruit, H. C., Nordlund, Å., \& Title, A. M. 1990, Annu. Rev. Astron. Astrophys. 28, 263

Steffen, M. 2007, in: F. Kupka, I. W. Roxburgh, \& K. L. Chan (eds.), Proc. IAU S 239, (Cambridge: Camb. Univ. Press), p. 36

Stein, R. F. \& Nordlund, Å. 1998, ApJ, 499, 914

Stix, M. 1989, The Sun (Berlin: Springer-Verlag)

Title, A. M. 2007, in: K.A. van der Hucht (ed.), Highlights of Astronomy, (Cambridge: Camb. Univ. Press), vol. 14, p. 30

Townsend, A. A. 1956, The structure of turbulent shear flow (Cambridge: Camb. Univ. Press)

Vögler, A., Shelyag, S., Schüssler, M., Cattaneo, F., Emonet, T., \& Linde, T. 2005, A\&A 429, 335 
Vögler, A. \& Schüssler, M. 2007, A\&广A, 465, L43

Wedemeyer, S., Freytag, B., Steffen, M., Ludwig, H.-G., \& Holweger, H. 2004, A\&A 414, 1121

Weiss, A., Hillebrandt, W., Thomas, H.-C., \& Ritter, H. 2004, Cox $E$ Giuli's Principles of Stellar Structure. Extended Second Edition, (Cambridge: Cambridge Scientific Publ.)

Xiong, D. R. 1978, Chinese Astronomy 2, 118

\section{Discussion}

K. Stepien: Have you tried to calculate acoustic energy flux heating the chromosphere?

F.KUPKA: Not yet, but we plan to do that and are looking forward to it.

P. Woitke: Concerning the "switch" between diffusion limits and bin-Z-dependent radiative transfer: (1) Why do you do that? (2) Do we have to worry about it? (3) What is your numerical recipe for this "switch"?

F. KupkA: (1) Saving computational time. (2) The bin-method is only good around $\tau=1$ where it is constructed for. Applying it to too deep layers leads actually to errors. (3) We apply a simple geometric criterion, namely a specified height, about half of the computational domain is diffusive transfer.

(H.-G. LudwiG: How far do we have to go in resolution in order to reach the regime where the turbulence properties in the models became really self-similar (or obey e.g., a Kolmogorov scaling law)?

F. KUPKA: I would say until we resolve shear driven turbulence so that its dynamics is captured on the computational grid, a Kolmogoro spectrum showing up at small scales should be a good indicator. We are currently performing simulations at even higher resolution to obtain further clues on that. 